\title{
A Comparison between independent living elders and residents in 25 German Nursing Homes following the Group-Living Principle. An empirical study focusing on the subjective perspective of quality of live
}

Anke Simon ( $\square$ anke.simon@dhbw-stuttgart.de)

Research

Keywords: Quality of life, empirical study, nursing homes, group-living principle

Posted Date: February 19th, 2020

DOI: https://doi.org/10.21203/rs.2.23999/v1

License: (c) (1) This work is licensed under a Creative Commons Attribution 4.0 International License. Read Full License 


\section{Abstract}

Objective. The purpose of this study was to analyse the subjective quality of life (QoL) perceived by older people in German nursing homes following the group-living principle.

Methods. The Nottingham Health Profile (NHP) as health-related QoL measuring instrument was employed in 25 nursing homes ( $\mathrm{n}=404$ participants). A comparison with the national German representative sub-sample of independent living elders (age-group over 75) was conducted. Psychometric properties and appropriateness were analysed.

Results. Our findings indicate an acceptable perception of residents' QoL. The mean NHP scale scores show, that the perceived QoL of residents in group-living nursing home reached nearly the same level as independently living elders (national German reference values, age group over 75 years, except for the NHP sub-scale physical mobility). QoL related results on life satisfaction and feeling of happiness confirm the NHP findings. The preliminary pilot study protocol could be replicated, according to good scientific practice.

Conclusions and implications. The report presents the first major investigation in the research field of subjective quality of life in group-living nursing homes. The study focused on people over 75 years of age with age-specific reduced physical and mental abilities. The NHP should also be considered as a reliable, valid and for older people appropriate instrument. Due to the lack of research on residents' perspectives, further studies should follow to establish age-specific and care setting-specific reference data for nursing home residents. In particular, more research is needed to answer the question, which care setting best meets people's essential needs in older age.

\section{Background}

Group-living care settings have evolved as a reaction to the critical public opinion regarding to the hospital-like environment of traditional nursing homes. The concept originated in Sweden, and was later introduced in countries, such as the Netherlands (Eerden \& Jones 2011), Great Britain (Lindesay et al. 1992), France (Ritchie et al. 1992), and Japan (Funaki et al. 2005). Although group-living, home-like nursing homes have been established as an alternative to nursing homes (settings) for many years, there is no generalised definition of the term "group-living nursing home", but common principles and concepts related to this model:

- Archetypical nursing homes consist of several group-living units with up to 15 residents living together in each unit.

- Nursing homes provide home-like environments as much as possible.

- Individual space, respectively a small private apartment or a one-room-unit is provided for each resident. Additional large dayrooms/lounge kitchen areas serve as shared space, where the majority of daytime is 
spent together with other residents.

- Vision of 'active aging', allowing as much privacy and independence as possible. Comparable to the residents' former homes no centralized food and no centralized washing service is provided.

- A mixed care team (an educated care-giver per unit, nursing staff and voluntaries per nursing home) compensate for declining ability and vitality, and lowers the burden for family care.

- Mix of residents (with psychogeriatric complaints, i.e. dementia, and with somatic, physiological ailments) live together.

Often, in public opinion conventional nursing homes are associated with single-sided thoughts, such as the last stage before death, poor quality of life (QoL) and loss of independency. The implemented German standard assessment instrument for external mandatory evaluation of nursing homes, based on the German Care Transparency Act (§ 115 Abs. 1a SGB XI), mainly focuses on the evaluation of objective quality indicators, mainly structure and process quality attributes. Critical discussions among health scientists, health professionals and health politicians about methodological issues and the lack of outcome criteria let to a recent revision, which will be introduced in December 2019 (Hassler \& WolfOstermann 2010; Möller \& Zieres 2010; Sünderkamp et al. 2014; Przylog et al. 2016; Wingenfeld \& Engels 2011). Although the newly revised German assessment instrument covers essential outcome quality criteria such as prevention of mobility loss, independent maintenance of daily activities, unintended weight loss; and solves most of the mentioned issues, the viewpoint of residents is still not included.

Current research proposes the implementation of quality of life measurements to cover residents' viewpoints on care (Becker et al. 2005; Brandenburg \& Günther 2015; de Boer et al. 2017; Grabowski et al. 2014; Hassler \& Wolf-Ostermann 2010; Kok et al. 2018; Palm et al. 2018; Vaarama et al. 2008). So far only a few studies investigating subjective quality of life in older people can be found. They mainly focus on older, multi-morbid patients without dementia (Hassler \& Wolf-Ostermann 2010; Holzhausen 2009; Warnke et al. 2004), address patients with dementia partly in special settings (Auer, et al. 2017; Ettema et al. 2005; Gertz \& Berwig 2008; Kok et al. 2018; Logsdon et al. 2002; Palm et al. 2018; Sixsmith et al. 2008), and consider care-related measures in nursing home residents (Becker et al. 2005; Schaal et al. 2015; Vaarama et al. 2008). According to our research there is still a complete lack of studies on groupliving nursing homes residents in Germany and elsewhere, apart from one preliminary pilot study published in 2013. In Simon et al. (2013), we researched certain quality of live measurements according to the following criteria: a) the scale dimensions include aspects of physical and mental well-being, social relations and daily life appropriate for the target group of nursing home residents (Rupprecht 2006), b) the scale has been validated as paper \& pencil questionnaire and face-to-face interview, c) nation-wide age-specific reference values are available. The selection process included generic profile-based instruments (i.e. Short Form Survey 36 / SF-36 and Nottingham Health Profile / NHP) and preferencebased measures with a single-dimension index score (i.e. Short Form Survey Six Dimension / SF-6D, Health Utility Index / HUI, EuroQol- 5 Dimension / EQ-5D). During the preliminary investigation in 2013 a 
questionnaire appropriate for elders could be validated. Descriptive findings in seven nursing homes indicated an acceptable quality of live.

The study presented here follows two objectives: Our main intention is to measure the residents' subjectively perceived quality of life in group-living nursing homes. The second aim of our investigation is to replicate our preliminary study results as well as the implemented research protocol (Simon et al. 2013) according to good scientific practice.

Quality of life was assessed by using the Nottingham Health Profile (NHS) (Hunt \& McEwen 1980). The $\mathrm{NHP}$ is one of the most commonly used generic scale for measuring health-related quality of life and has already been used in nursing home studies with frail elders (Noro 1998; Warnke et al. 2004). The 38 items, formulated as direct statements, aim to identify self-assessed QoL impairments in six dimensions: physical mobility, pain, sleep, social isolation, emotional reaction, and energy level. Gunzelmann et al. (2006) confirmed the psychometric validation of the German NHP version (Kohlmann et al.1997). The authors provided age-specific reference values as representative sub-sample.

\section{Methods}

Participants and data collection

The exploratory cross-sectional study includes 25 group-living nursing homes in Germany and was conducted in January 2018. The care provider (private, non-profit organisation) is one of the first, introducing group-living nursing homes in Germany. All 25 nursing homes of our sample homogenously follow the cooperate group living policy (as mentioned before). Therefore, the investigation was conducted in a standardised setting, regarding aspects like homogenous philosophy of care, similar building features and domestic characteristics, 10 up to 14 residents per unit, standardised care concept and qualification mix of staff.

According to our study protocol (Simon et al. 2013), the inclusion criteria for selecting residents were as follows: living in nursing homes for more than three months, willing, and physically and mentally able to participate in the study. Residents with cognitive impairment, i.e. dementia, were not excluded for ethical reasons. Naturally, many of the residents in nursing homes suffer from various stages of dementia in different stages. They do however have the human right to freedom of expression. Nevertheless, nursing homes head nurses pre-selected the target population regarding the general ability to understand and answer quality of life and health-related questions. As proposed in the preliminary pilot study, patients with severe depressive symptoms were excluded (Simon et al. 2013).

Data acquisition was accomplished by face-to-face interviews. Due to the special setting of nursing homes with bodily and cognitively impaired older people (including residents with dementia), we replicated our study design's introduction procedure (Simon et al. 2013), comprising four well-prepared steps: (1) all investigators underwent a short training on qualitative research methods and the interview guideline; (2) organized groups (two to three interviewers assigned to each nursing home) visited the 
related group-living units during two days; (3) to get in naturally contact with the residents the investigators were introduced to the residents by the head care-giver very early with the start of the morning shift, i.e. they helped prepare breakfast and ate with the elders, assisted with the morning toilette and bed making; (4) the investigators spent the entire two days together with the nursing home residents to create a familiar atmosphere. Thereby, time pressure and uncomfortable situations could mainly be avoided. Nursing home residents and investigator decided freely when and where to get through the interview. Length of the interview was not limited. The investigators were allowed to explain the statements and scales if necessary or read them aloud.

\section{Questionnaire}

The measurement tool was administered as self-reported questionnaire (Simon et al. 2013). The NHP (Hunt \& McEwen 1980) was used in a German version validated by Kohlmann et al. (1997) and by Gunzelmann et al. (2006) for the population of independently living people. The scale consists of 38 QoL items, related to six dimensions, using a binary (yes or no) scale. Residents confirmed each statement with "yes" (when there was a complaint or handicap, computed as 1) and denied with "no" (when there was no complaint or handicap, computed as 0 ) regarding his or her (living) situation at the time of the interview. Following the calculation instruction (Kohlmann et al. 1997), each 'yes' was weighed according to its importance in the dimension and scored between 0 (maximum quality of life) and 100 (no quality of life). The dimensions' score was not calculated when the resident was unable to completely respond to the related items.

To compare our obtained data with the German reference values, we used a representative sub-sample of independent living people older than 75 years (Gunzelmann et al. 2006; Simon et al. 2013). Furthermore, we added two QoL related scales to the original pilot study protocol: the OECD life satisfaction scale (2017; single item scale from minimum score 0 to maximum score 10) and the feeling of happiness scale by Inglehart et al. (2014; Likert scale from 1 - not happy at all to 4 - very happy) and compared our findings with reference data provided by the OECD Better Life Index and the World Value Survey.

Following the original pilot study protocol, we included socio-demographic and health-related variables: age, sex, marital status, lengths of stay, current state of health (five-point Likert scale from 1 - very poor to 5 - very good), grade of dependency (German assessment standard Pflegegrad) and the AMT4 (shortened version of the Abbreviated Mental Test by Swain and Nightingale (1997)).

Statistical analysis

The Statistical Package for the Social Sciences (SPSS), version 23, was used for all analyses. The descriptive results comprise mean, standard deviation, frequency and percentage. Possible differences between the sub-sample residents older than 75 years and the German reference data of independently living older people were gender-specifically verified using the t-test. The NHP reliability analyses (internal consistency, split-half reliability) and the inter-correlation of the scales were replicated and reported according to our pilot study protocol (Simon et al. 2013). Significance was set at $5 \%$ level $(p<0.05)$. 


\section{Results}

Study population

Out of a total of 1577 residents in 25 nursing homes, 615 were physically and cognitively able to answer the questionnaire. 404 residents participated in the study (response rate 66\%). Reasons for declining were mainly due to a lack of interest, not enough time, and absence during the time of interviews. Table 1 shows the baseline characteristics of the participating residents. 78.5 percent of the participants were female; the mean age was 84.6; the majority were widowed (76.7\%). Approximately two third of the respondents have lived in the nursing homes for more than one year. 33.4 percent were tested with an impaired cognitive status. Most participants have been categorised in grade 2 or 3 of dependency.

\section{Quality of life}

Firstly, the mean NHP score over all scales (166.54, SD 94.04), and secondly the mean scores of the six different NHP scales were calculated. Figure 1 shows the nursing home residents' subjective quality of life scores between 0 (maximum quality of life) and 100 (no quality of life), means the lower the NHP score the higher the perceived quality of life. Regarding figure 1, the NHP scale social isolation scores lowest (15.49) followed by the sub-scale emotional reaction (17.24) indicating residents' high quality of life on those areas. On the contrary, the NHP scale physical mobility with the highest score (45.66) was perceived as the worst subjective QoL area. The NHP scales pain, sleep and energy loss show a score level in between the previous results. Thirdly, we compared our findings with German reference group over 75 years, drawn from the above mentioned NHP national dataset. The national reference data set included only older people living independently (the sample does not include older people living in an institution). The comparison findings are shown in Table 2. The t-test identified only a few significant differences between elders living independently and group-living nursing home residents. The highest disparity can be detected in physical mobility. Nursing home residents are more affected with physical impairments and loss of mobility (mean score female 46.02, mean score male 44.17) than independently living older people (mean score female 27.01, mean score male 22.22). Women in nursing homes score higher on pain (mean 27.78 versus 21.41 ) and on sleep than women living independently (mean 33.80 versus 27.90). No significant differences can be encountered with the NHP scale energy loss. For both genders, minor differences were found on the emotional reaction scale and on men's social isolation perception. The sub-sample of German reference data of independently living elders shows moderate gender differences, as do our findings presented here. In accordance with our NHP results, QoL related scale scores on life satisfaction and happiness reached the German reference levels only with slight differences (below as well as above).

Reliability of the NHP in group-living nursing home residents

Table 3 shows the mean and standard deviation as well as Cronbach's alpha and the split-half-reliability of the NHP for the 404 home residents. All scales can be regarded as sufficient as we obtained high values regarding Cronbach's alpha and split-half reliability. 
Table 4 shows inter-correlations for all NHP scales. In general, positive correlations appear between all NHP scales. The highest inter-correlations were found between physical mobility and pain, and between emotional reaction and energy loss.

Correlation analysis shows a statistically significant moderate reverse relation between the NHP score and the currently perceived status of health $(r-.283, p \leq .01)$. The highest correlation was found between the perceived status of health and the NHP scale pain $(r-.272, p \leq .01)$, followed by the NHP scale physical mobility $(r-.256, p \leq .01)$. The NHP score correlates also positively with residents's feeling of happiness ( $r .295, p \leq .01)$ and life satisfaction ( $r .393, p \leq .01)$. No statistically significant relation can be found between NHP score and age. Residents with cognitive impairments (positive AMT4 test) generally estimated their quality of life higher than others (NHP score $135.48, p \leq .001$, eta .243 / eta ${ }^{2}$ .059).

\section{Discussion And Limitation}

The present study describes the subjective quality of life in group-living nursing home residents. Homelike living, social interaction and domestic activities are important characteristics of group-living nursing homes. According to our main objective, the present study conducted face-to-face interviews employing the Nottingham Health Profile (NHP). The mean NHP scale score over all dimensions suggests an adequate perception of quality of life. Among the five different NHP scales, social life and emotional wellbeing scored best regarding subjective quality of life, whereas physical mobility showed the lowest quality perception. We regard deteriorated physical mobility as one of the main reasons for living in a nursing home. Several other studies came up with similar findings investigating mobility in older people (Wirtz et al. 2018; Yümin et al. 2011). The comparison with reference values of independently living older people in Germany revealed further results. Although a few significant differences between the two subsamples of persons older than 75 years could be found, our findings indicate that with exception of the NHP scale physical mobility, the perceived quality of life of group-living nursing home residents and independently living elders over 75 years are nearly on the same level. Accordingly, our present findings in 25 nursing homes confirm those of the preliminary pilot study conducted in seven nursing homes in 2013. Further QoL-related scales, specifically residents' perceived life satisfaction and feeling of happiness indorse the NHP results.

Former study findings, although not directly related to group-living settings, indicate that active conduct of live and social interactions in older life have a decisive impact on QoL (Graefe et al. 2011; Livingston et al. 2017; Xu et al. 2019).

Subjective quality of life is an essential criterion for the evaluation of nursing homes and geriatric care. Furthermore, patient-outcome research on the (assumed) value of innovative care models and 'new living and care arrangements', such as group-living principles substituting former institutional care, makes an important resource for health policy-makers and public health researchers. Next to objective quality indicators, appropriate measurements of residents' perspectives are necessary to comprehensively 
evaluate the impact of innovative home care settings. Hence, our second objective was to replicate the previous study protocol aiming to validate the scales implemented at the time. A recent meta-analysis by Camerer et al. (2018) revealed that many studies in social sciences could not be replicated (replication crisis). The verification of study designs assumes a very important part of good scientific practice. However, the psychometric quality of the NHP instrument turned out to be sufficient (internal consistency and split-half-reliability were satisfactory). The questionnaire was appropriate for nursing home residents with age-specific mental and functional impairments. The NHP sub-scales show various intercorrelations. Consistent with our pilot study design (Simon et al. 2013) and with the findings from the nation-wide sample of independently living people (Gunzelmann et al. 2006), we found a relatively high correlation between physical mobility and pain. As shown in the same study, we could also replicate significant correlations between energy loss, emotional reaction and social isolation, suggesting that energy loss does not only result from physiological aging but can also be understood as linked to social and emotional well-being.

In spite of the common assumption that nursing home residents are unable to understand and answer complex questions properly (Hassler \& Wolf-Ostermann 2010), the participants of our study responded well to the interviews. The majority of NHP items were good to understand, as the two response choices (yes or no) were easy to handle for the interviewees. Naturally, many nursing home residents find it hard to manage paper \& pencil tests due to impaired vision or hearing, difficulties concentrating and other dementia related ailments. Reading the questionnaire loud in in a face-to-face interview proved to be most practical. Also, we could avoid the supportive assistance of nursing home staff (often required with self-administered paper \& pencil questionnaires), to prevent possible biases on the effect of socialdesirability-responses.

On average the interview was completed within 40 minutes. However, some interviews took about two hours because some residents found pleasure in telling about their lives.

The negatively formulated NHP statements worried some participants. In these cases, interviewees received close attention and further explanations.

Naturally, the cross-sectional design of the present study (at a specific point in time) exhibits some limitations. The preselection of the samples according to residents' eligibility may limit the subsequent interpretation as well as the relevance of the data. Also, the fact that personal characteristics of residents in group-living settings might differ from those of regular nursing home residents limits the comparability of the data.

The Nottingham Health Profile is one of the most frequently used generic instruments in the area of health-related quality of life measurements. It appears to be particularly appropriate for the questioning of older people as the items are short and answering is relatively easy. Nevertheless, the NHP concept focuses generally on negative criteria in measuring subjective quality of life, albeit from a holistic point of view, positive aspects could be an enrichment. Moreover, some items in the dimension social isolation might trigger relatively strong emotional reactions (with regard to the findings in our pilot study in 2013) 
and reported by Warnke et al. 2004). Accordingly, the NHP scale, and particularly the subscale social isolation, is not recommendable for evaluating subjective quality of live in residents with depressive symptoms, as they are often related to dementia.

Although the NHP covers areas of particular importance to older people, such as loss of energy, pain, sleeping disorders, social isolation, mobility and emotional health it might be useful to enhance the instrument with subscales focussing on specific QoL aspects, which are related to nursing care settings (Xu et al. 2019).

\section{Conclusion And Implications}

To our knowledge this investigation provides unique evidence about the subjective quality of life in groupliving nursing homes. The study, presented here, (1) addresses very old people with age-specific reduced physical and mental abilities; (2) includes cognitively impaired participants; and (3) encompasses with 25 nursing homes the first major study in the research field of subjective quality of life in German groupliving nursing homes. Moreover, the previous pilot study protocol and the preliminary results could be successfully replicated.

The presented findings intend to start a discussion: in many countries, traditional hospital-like nursing homes are evolving. Instead, new initiates aim at improving the notion of nursing home care to better meet people's needs, improve the quality of care and eventually enhance quality of life. To varying degrees, many of these activities focus on aspects of home-like nursing environments, such as traditional nursing home with enhanced activities, group-living nursing home, combination care home (residential and nursing home under one roof), small group nursing home, nursing home in rural farm settings etc.

Therefore, we recommended a nationwide scientific evaluation program on subjective perceived outcome quality to provide representative preference values and to compare various care settings.

Due to the lack of research on residents' perspectives on QoL, further studies should follow to establish age-specific and care setting-specific reference data for nursing home residents. In particular, more research is required to answer the question of which care setting meets best the essential needs of older people. We welcome participatory efforts.

\section{Abbreviations}

Diff Difference between mean values, EL Energy loss, ER Emotional reaction, EQ5D EuroQol- 5 Dimension, $f$ Female, $\mathrm{FH} \quad$ Feeling of happiness, $\mathrm{HUI} \quad$ Health utility index, LS Life satisfaction, $M \quad$ Mean value, $m$ Male, NHP Nottingham Health Profile, OECD Organisation for Economic Cooperation and Development, P Pain, PM Physical mobility, QoL Quality of Life, S Sleep, SD Standard deviation, SF-36 Short form survey 36, SF-6D Short-form survey six-dimension, SH Status of Health, SI Social isolation, a Internal consistency (Cronbach's alpha), rtt Split-half-reliability (Spearman-Brown) 


\section{Declarations}

\section{Acknowledgements}

We thank the participating residents and nursing staff of 25 nursing homes. We are also indebted to the students (school of Health Sciences \& Management, year 2015) for helping with the field work. Kaspar Pfister and Michael Barkow have provided administrative support, and we thank them all.

\section{Authors' contributions}

AS designed the study, carried out the study, performed statistical analysis and wrote the manuscript.

\section{Ethical approval and consent to participate}

The study was conducted in full accordance to the ethical standards of the institutional and national research committee and to the Would Medical Association Declaration of Helsinki, the guidelines and recommendations for ensuring Good Epidemiological Practice (GEP), and the international code for market and social research (ICC/ESOMAR). Informed consent was obtained from all individual participants included in the study.

\section{Funding}

The study was supported by a grand for travel and analysis expenditures from the nursing home management organization (non-profit company).

\section{Availability of supporting data}

Please contact author for data requests.

\section{Consent for publication}

Not applicable.

\section{Competing interests}

There is no personal or financial conflict of interest. The study was supported by a grand for travel and analysis expenditures from the nursing home management organization (non-profit company). The sponsor had no role in study design, data collection and analysis, decision to publish, or preparation of the manuscript.

\section{References}

Auer S, Kienberger U, Pascher P, Geck M, Hofmann B, Viereck C \& Span E (2017) Small scale group living versus traditional nursing home for persons with dementia. Pflegewissenschaft 19:156-165 
Becker S, Kruse A, Schröder J \& Seid IU (2005) Das Heidelberger Instrument zur Erfassung von Lebensqualität bei Demenz (H.I.L.DE.). Z Gerontol Geriat 38:108-121

Brandenburg H \& Günther H (2015) Gerontologische Pflege. Hogrefe, Bern

Camerer C, Dreber A, Holzmeister F, Ho T-H, Huber J, Johannesson M, Nosek BA (2018) Evaluating the replicability of social science experiments in Nature and Science between 2010 and 2015. Nature Human Behaviour. doi:http://dx.doi.org/10.1038/s41562-018-0399-z

de Boer B, Hamers JP, Zwakhalen SM, Tan FE \& Verbeek H (2017) Quality of care and quality of life of people with dementia living at green care farms: a cross-sectional study. BMC geriatrics 17 (1):155

Eerden van der WJ \& Jones GM (2011) Dutch large-scale dementia-care environments: a village within the community. Care Services Management 5 (3):137-146

Ettema TP, Droes R-M, de Lange J, Mellenbergh G J \& Ribbe MW (2005) A review of quality of life instruments used in dementia. Qual Life Res 14 (3):675-686

Funaki Y, Kaneko F \& Okamura H (2005) Study on factors associated with changes in quality of life of demented elderly persons in group homes. Scand J Occup Ther 12:4-9

Gertz HJ \& Berwig M (2008) Ist die Lebensqualität von Demenzpatienten messbar? . Nervenarzt 79:10231035

Grabowski DC, O'Malley AJ, Afendulis CC, Caudry DJ, Elliot A \& Zimmerman S (2014) Culture change and nursing home quality of care. Gerontologist. 54 Suppl 1:535-545

Graefe S, van Dyk S \& Lessenich S (2011) Being old is occurring later. Age-related norms and selfconcepts in the second half of life. Z Gerontol Geriat 44:299-305

Gunzelmann T, Hinz A \& Brähler E (2006) Subjective health in older people. GMS Psycho-Social-Medicine $3: 1-10$

Hassler M \& Wolf-Ostermann K (2010). Wissenschaftliche Evaluation zur Beurteilung der PflegeTransparenzvereinbarungen für den ambulanten (PTVA) und stationären (PTVS) Bereich. www.gkvspitzenverband.de, Accessed 25 September 2018

Holzhausen M (2009) Lebensqualität älterer Menschen. Konstruktion eines neuen individualisierten Messverfahrens. Hans Huber, Bern

Hunt SM \& McEwen J (1980) The development of a subjective health indicator. Sociol Health Illn 2:231246

Inglehart R, Haerpfer C, Moreno A, Welzel C, Kizilova K, Diez-Medrano J, Puranen B (2014) World Values Survey: Round Six - Country-Pooled Datafile Version. 
http://www.worldvaluessurvey.org/WVSDocumentationWV6.jsp, Accessed 25 September 2018

Kohlmann T, Bullinger M \& Kirchberger-Blumstein I (1997) The German version of the Nothingham Health Profile (NHP). Methodological issues of translation and psychometric validation. Soz Präventivmed 42:175-185

Kok JS, Nielen MM \& Scherder EJ (2018) Quality of life in small-scaled homelike nursing homes: an 8month controlled trial. Health and quality of life outcomes 16 (1):38

Lindesay J, Briggs K, Laves M, Macdonald A \& Herzberg J (1992) The Domus philosophy: a comparative evaluation of a new approach to residential care for the demented elderly. Int J Geriatr Psychiatry 6 (10):727-36

Livingston G, Sommerlad A, Orgeta V, Costafreda SG, Huntley J, Ames D, Schn (2017) Dementia prevention, intervention, and care. Lancet 390(10113):2673-2734

Logsdon R, Gibbons LE, McCurry S M \& Teri L (2002). Assessing quality of life in older adults with cognitive impairment. Psychosom Med 64: 510-519

Möller J \& Zieres G (2010) Zur Transparenz der Qualität von Pflegeeinrichtungen. On quality and transparency of care facilities. Gesundh ökon Qual manag 15:39-44

Möller J, Panhorst H \& Zieres G (2010) Qualitätsberichterstattung für ambulante Pflegedienste. Anspruch und Realität. Public reporting on professional nursing home care demand and reality. Gesundh ökon Qual manag 15:185-191

Noro A (1998) Long-term institutional among Finnish elderly population: trend and potential discharge. Stakes, Helsinki

OECD (2017) Better Life Index. www.oecdbetterlifeindex.org, Accessed 25 September 2018

Oswald WD, Lehr U, Sieber C \& Kornhuber J (2006) Gerontologie. Medizinische, psychologische und sozialwissenschaftliche Grundbegriffe. Kohlhammer, Stuttgart

Palm R, Trutschel D, Sorg CG, Dichter MN, Haastert B \& Holle B (2018) Quality of Life in People With Severe Dementia and Its Association With the Environment in Nursing Homes: An Observational Study . Gerontologist 59:665-674

Przylog A, Stroka MA, Engel S \& Linder R (2016) Bieten Pflegeheime mit besseren Pflegenoten auch eine bessere Qualität? Empirische Analyse auf Basis von GKV-Routinedaten. Z Gerontol Geriat 49:308-316

Ritchie J, Colvez A, Ankri J, Ledesert B, Gardent H \& Fontaine A (1992) The evaluation of long-term care for the dementing elderly: a comparative study of hospital and collective non-medical care in France. Int $J$ Geriatr Psychiatry 7 (8):549-557 
Rupprecht R (2006). Lebensqualität. In: Oswald WD et al (Ed) Gerontologie. Medizinische, psychologische und sozialwissenschaftliche Grundbegriffe. Kohlhammer, Stuttgart, pp 242-247

Sünderkamp S, Weiß C \& Rothgang H (2014) Analyse der ambulanten und stationären Pflegenoten hinsichtlich der Nützlichkeit für den Verbraucher. Pflege 27 (5):325-336

Schaal T, Schönfelder T, Klewer J \& Kugler J (2015) Quality of care in German nursing homes. A longitudinal study regarding quality assenssments from 2009 to 2013. HeilberufeScience 6:3-9

Sharples LD, Todd CJ, Caine N \& Tait S (2000) Measurement properties of the Nottingham Health Profile and Short Form 36 health status measures in a population sample of elderly people living at home: Results from ELPHS. Br J Health Psychol 5:217-233

Simon A, Berwig M \& Heinrich S (2013) Quality of life in Nursing Homes following the Group-Living Principle. A pilot study measuring the quality of life from the subjective perspective of dependent-living elderly. Z Gerontol Geriat 46:556-562

Sixsmith A, Hammond M \& Gibson G (2008) Quality of life and dementia. In: Vaarama M, Pieper R, Sixsmith A (ed) Care-related quality of life in old age: concepts, models, and empirical findings. Springer, New York, pp 217-233

Swain DG \& Nightingale PG (1997) Evaluation of a shortened version of the Abbreviated Mental Test in a series of elderly patients. Clin Rehabil. 11 (3):243-248

Vaarama M, Pieper R \& Sixsmith A (2008) Care-related quality of life in old age: concepts, models, and empirical findings. Springer, New York

Vaarama M, Pieper R \& Sixsmith A (2008) The general framework and methods for the care keys research. In: Vaarama M, Pieper R, Sixsmith A (ed) Care-related quality of life in old age: concepts, models, and empirical findings. Springer, New York, pp 3-18)

Warnke A, Meyer G, Bott U \& Muelhausen I (2004) Validation of a quality of life questionnaire measuring the subjective fear of falling in nursing home residents. Z Geront Geriat 37: 459-466

Wingenfeld K \& Engels D (2011) Entwicklung und Erprobung von Instrumenten zur Beurteilung der Ergebnisqualität in der stationären Altenhilfe. Abschlussbericht. Bielefeld, Bundesministeriums für Gesundheit und des Bundesministeriums für Familie, Senioren, Frauen und Jugend. IPW, Köln

Wirtz MA, Morfeld M, Brähler E \& Glaesmer H (2018) Association of Physical Morbidity and HealthRelated Quality of Life in a Representative Sample of Older German People. European Journal of Health Psychology 25(4):140-151

Xu D, Mou H, Gao JXW, Ling J \& Wang K (2019) Quality of life of nursing home residents in mainland China: The role of children and family support. Arch Gerontol Geriatr 83:303-308 
Yümin ET, Simsek TT, Sertel M, Öztürk A \& Yümin M (2011) The effect of functional mobility and balance on health-related quality of life (HRQoL) among elderly people living at home and those living in nursing home. Arch Gerontol Geriatr 52:180-184

\section{Tables}

Table 1 Baseline characteristics of participants

\begin{tabular}{|c|c|c|}
\hline Characteristics & & Participants \\
\hline \multirow[t]{2}{*}{ Gender } & male & 87 (21.5\%) \\
\hline & female & 317 (78.5\%) \\
\hline \multirow[t]{3}{*}{ Age } & average & 84.6 \\
\hline & standard deviation & 7.8 \\
\hline & span & $47-102$ \\
\hline \multirow[t]{6}{*}{ Marital status } & married/living together & $39(9.7 \%)$ \\
\hline & married/living apart & $2(0.5 \%)$ \\
\hline & single & $37(9.2 \%)$ \\
\hline & divorced & $11(2.7 \%)$ \\
\hline & widowed & $310(76.7 \%)$ \\
\hline & not available & $5(1.2 \%)$ \\
\hline \multirow[t]{3}{*}{ Length of stay } & $<1$ year & $146(36.1 \%)$ \\
\hline & $1-2$ years & $101(25.0 \%)$ \\
\hline & $>2$ years & 157 (38.9\%) \\
\hline \multirow[t]{7}{*}{ Grade of dependency } & non & $19(4.7 \%)$ \\
\hline & grade 1 & $6(1.5 \%)$ \\
\hline & grade 2 & $153(37.9 \%)$ \\
\hline & grade 3 & $172(42.6 \%)$ \\
\hline & grade 4 & 48 (11.9\%) \\
\hline & grade 5 & $3(0.7 \%)$ \\
\hline & not available & $3(0.7 \%)$ \\
\hline \multirow[t]{2}{*}{ cognitive status } & not impaired & $269(66.6 \%)$ \\
\hline & impaired & $135(33.4 \%)$ \\
\hline
\end{tabular}

Table 2 Comparison between group-living nursing home residents and German reference values over 75 years 


\begin{tabular}{|c|c|c|c|c|c|c|c|c|c|c|}
\hline \multirow[t]{2}{*}{ NHP Scales } & & \multicolumn{3}{|c|}{$\begin{array}{l}\text { Group-living } \\
\text { nursing home residents }\end{array}$} & \multicolumn{3}{|c|}{$\begin{array}{l}\text { German reference values } \\
\text { old people living independently }\end{array}$} & \multirow[t]{2}{*}{ Diff } & \multicolumn{2}{|l|}{$\mathrm{t}$} \\
\hline & & $\mathrm{M}$ & $\mathrm{SD}$ & $\mathrm{n}$ & M & SD & $\mathrm{n}$ & & & \\
\hline \multirow[t]{2}{*}{ Energy loss } & $\mathrm{m}$ & 28.12 & 28.62 & 64 & 33.33 & 40.82 & 45 & -5.21 & -1.359 & \\
\hline & $\mathrm{f}$ & 34.27 & 29.64 & 284 & 34.98 & 36.86 & 81 & -0.71 & -.485 & \\
\hline \multirow[t]{2}{*}{ Pain } & $\mathrm{m}$ & 22.92 & 26.26 & 60 & 21.94 & 29.33 & 45 & 0.98 & .229 & \\
\hline & $\mathrm{f}$ & 27.78 & 27.80 & 252 & 21.14 & 27.92 & 81 & 6.64 & 3.688 & $* * *$ \\
\hline \multirow[t]{2}{*}{ Emotional reaction } & $\mathrm{m}$ & 17.19 & 15.95 & 64 & 11.11 & 21.32 & 45 & 6.08 & 2.945 & $* *$ \\
\hline & $\mathrm{f}$ & 16.94 & 17.18 & 284 & 14.04 & 22.04 & 81 & 2.90 & 2.556 & $*$ \\
\hline \multirow[t]{2}{*}{ Sleep } & $\mathrm{m}$ & 34.69 & 25.76 & 64 & 31.56 & 32.89 & 45 & 3.13 & .671 & \\
\hline & $\mathrm{f}$ & 33.80 & 26.28 & 284 & 27.90 & 31.25 & 81 & 5.90 & 3.749 & $* * *$ \\
\hline \multirow[t]{2}{*}{ Social isolation } & $\mathrm{m}$ & 16.88 & 20.84 & 64 & 8.89 & 17.35 & 45 & 7.99 & 2.863 & $* *$ \\
\hline & $\mathrm{f}$ & 14.79 & 20.20 & 284 & 12.59 & 18.01 & 81 & 2,20 & 1.739 & \\
\hline \multirow[t]{2}{*}{ Physical mobility } & $\mathrm{m}$ & 44.17 & 22.25 & 60 & 22.22 & 27.94 & 45 & 21.95 & 6.814 & $* * *$ \\
\hline & $\mathrm{f}$ & 46.02 & 21.85 & 281 & 27.01 & 27.84 & 81 & 19.01 & 13.648 & $* * *$ \\
\hline
\end{tabular}

M mean, SD standard deviation, Diff difference between mean values, $m$ male, $\mathrm{f}$ female

$* * * \mathrm{p} \leq 0.001$ (2-sided) $* * \mathrm{p} \leq 0.01$ (2-sided) $* \mathrm{p} \leq 0.05$ (2-sided)

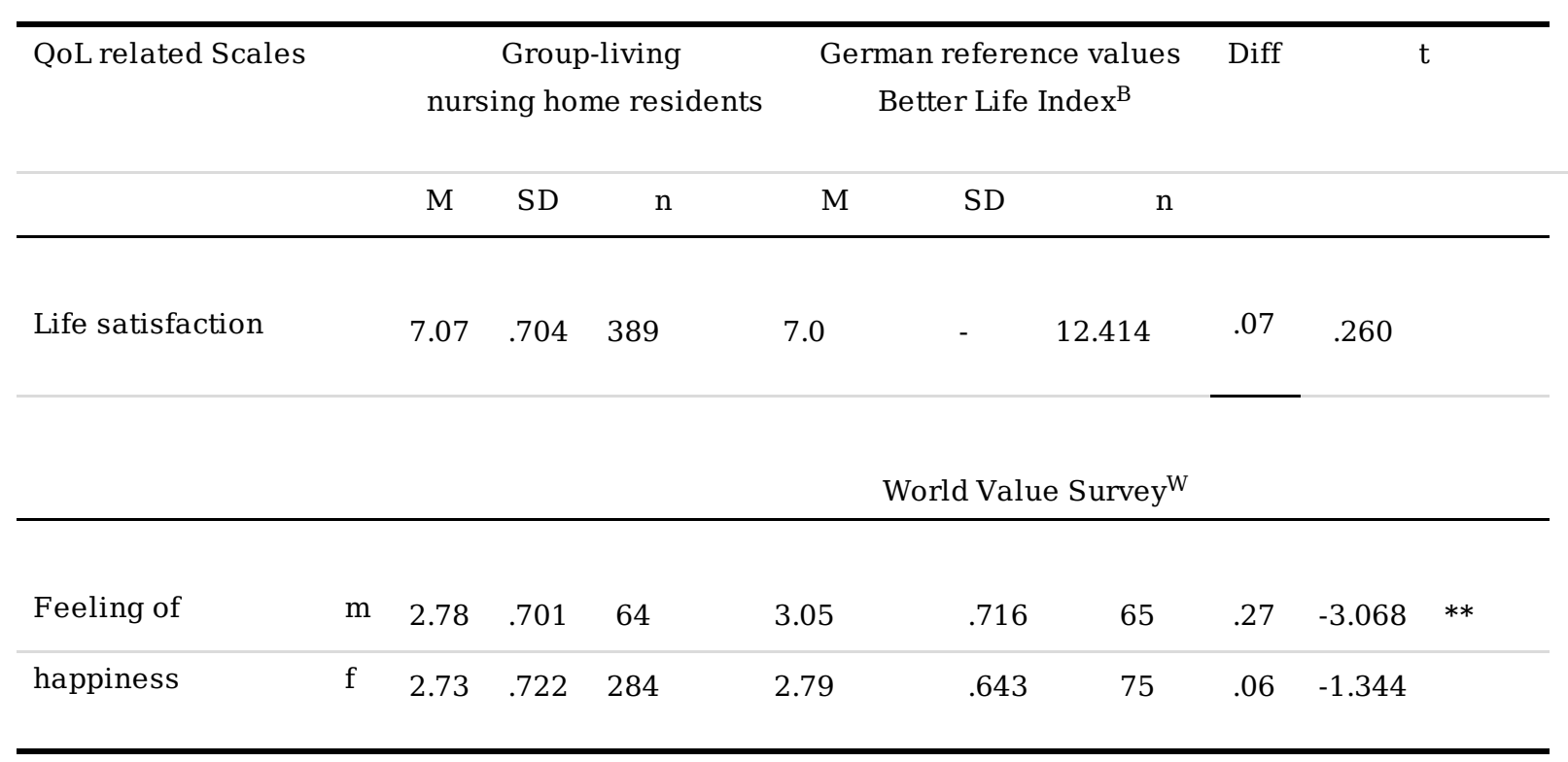

M mean, SD standard deviation, Diff difference between mean values 
$* * * \mathrm{p} \leq 0.001(2$-sided) $* * \mathrm{p} \leq 0.01$ (2-sided) $* \mathrm{p} \leq 0.05$ (2-sided)

${ }^{\text {B }}$ gender differentiation and SD not available ${ }^{33}$

W age group over 75 years selected and calculated based on the World Value Survey SPSS data set ${ }^{34}$

Table 3 Psychometric data for the items of the NHP for group-living elders

\begin{tabular}{lllll}
\hline Scale & $\mathrm{M}$ & $\mathrm{SD}$ & $\alpha$ & $\mathrm{r}_{\mathrm{tt}}$ \\
\hline Energy loss & 32.74 & 29.80 & 0.84 & 0.83 \\
Pain & 26.78 & 27.37 & 0.90 & 0.96 \\
\hline Emotional reaction & 17.24 & 17.44 & 0.76 & 0.80 \\
\hline Sleep & 34.46 & 26.17 & 0.84 & 0.85 \\
\hline Social isolation & 15.49 & 20.64 & 0.77 & 0.83 \\
\hline Physical mobility & 45.66 & 22.57 & 0.82 & 0.93 \\
\hline
\end{tabular}

M mean, SD standard deviation, $\alpha$ internal consistency (Cronbach's alpha), $\mathrm{r}_{\mathrm{tt}}$ split-half-reliability (Spearman-Brown)

Table 4 Inter-correlations of NHP scales and further relations

\begin{tabular}{|c|c|c|c|c|c|c|c|c|c|}
\hline & EL & $\mathrm{P}$ & ER & $\mathrm{S}$ & SI & $\mathrm{PM}$ & $\mathrm{SH}$ & LS & FH \\
\hline Energy loss (EL) & & & & & & & $-.193^{* *}$ & $-.269^{* *}$ & $-.236^{* *}$ \\
\hline Pain $(\mathrm{P})$ & $.329^{* *}$ & & & & & & $-.272^{* *}$ & $-.191^{* *}$ & $-.132^{*}$ \\
\hline Emotional reaction (ER) & $.399^{* *}$ & $.272^{* *}$ & & & & & $-.187^{* *}$ & $-.462^{* *}$ & $-.374^{* *}$ \\
\hline Sleep (S) & $.277^{* *}$ & $.370^{* *}$ & $.364^{* *}$ & & & & $-.146^{* *}$ & $-.214^{* *}$ & $-.110^{*}$ \\
\hline Social isolation (SI) & $.263^{* *}$ & $.171^{* *}$ & $.445^{* *}$ & $.218^{* *}$ & & & $-.208^{* *}$ & $-.386^{* *}$ & $-.329^{* *}$ \\
\hline Physical mobility (PM) & $.438^{* *}$ & $.457^{* *}$ & $.182^{* *}$ & $.265^{* *}$ & $.179^{* *}$ & & $-.256^{* *}$ & $-.178^{* *}$ & $-.113^{*}$ \\
\hline
\end{tabular}

$* * \mathrm{p} \leq .01(2$-sided $) * \mathrm{p} \leq .05$ (2-sided)

SH Status of health, LS Life satisfaction, FH Feeling of happiness

Figures 


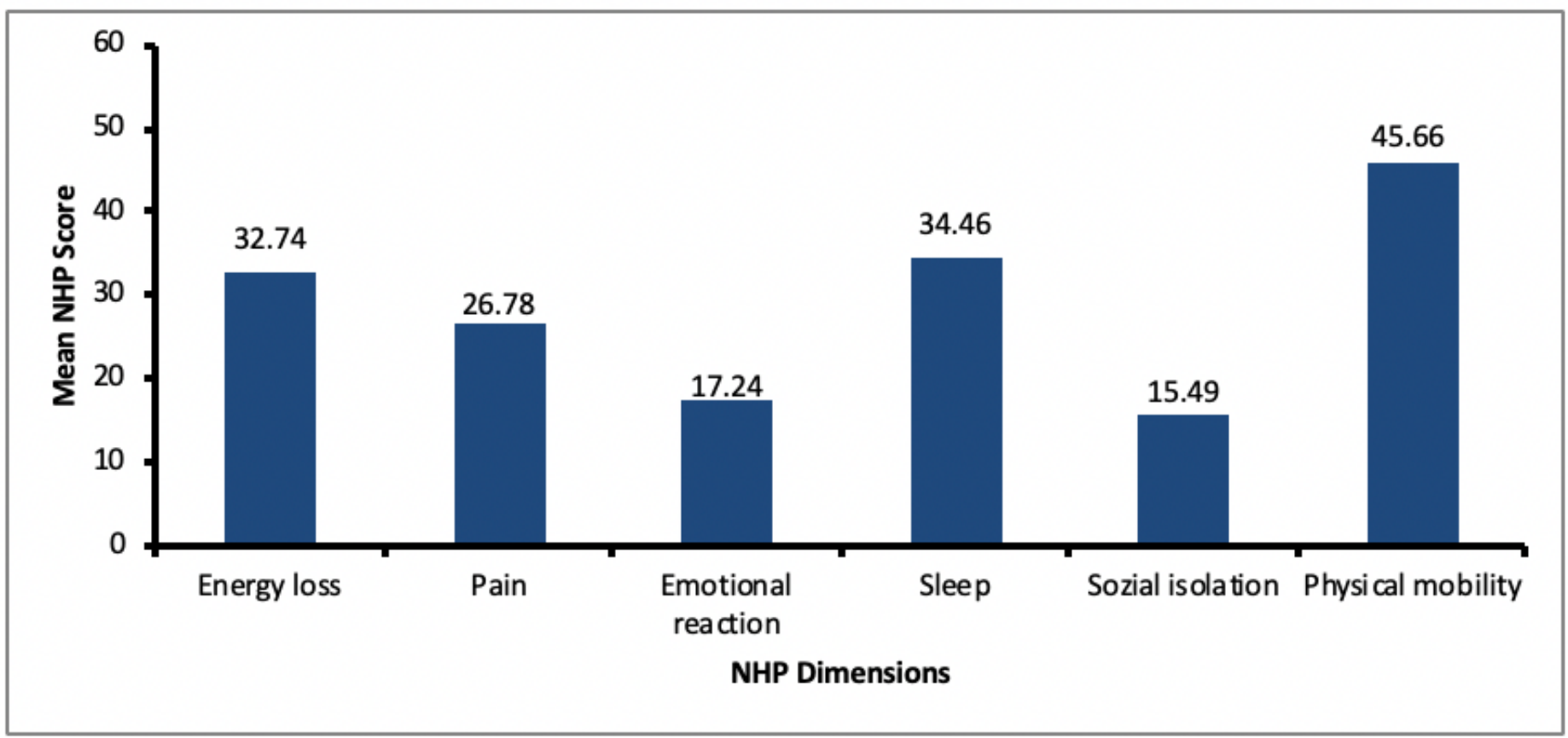

Figure 1

Descriptive results of the NHP scales

\section{Supplementary Files}

This is a list of supplementary files associated with this preprint. Click to download.

- DatasetEJAQoLnhgrouplivingprinciple.sav 